PUPT-1613

Imperial/TP/95-96/39

hep-th/9604089

\title{
Entropy of Near-Extremal Black p-branes
}

\author{
I.R. Klebanov* \\ Joseph Henry Laboratories \\ Princeton Unversity, Princeton, NJ 08544 \\ and \\ A.A. Tseytlin* \\ Theoretical Physics Group, Blackett Laboratory, \\ Imperial College, London SW' 2BZ, U.K.
}

\begin{abstract}
We carry out a thorough survey of entropy for a large class of $p$-branes in various dimensions. We find that the Bekenstein-Hawking entropy may be given a simple world volume interpretation only for the non-dilatonic $p$-branes, those with the dilaton constant throughout spacetime. The entropy of extremal non-dilatonic $p$-branes is non-vanishing only for solutions preserving $1 / 8$ of the original supersymmetries. Upon toroidal compactification these reduce to dyonic black holes in 4 and 5 dimensions. For the self-dual string in 6 dimensions, which preserves $1 / 4$ of the original supersymmetries, the near-extremal entropy is found to agree with a world sheet calculation, in support of the existing literature. The remaining 3 interesting cases preserve $1 / 2$ of the original supersymmetries. These are the self-dual 3-brane in 10 dimensions, and the 2- and 5-branes in 11 dimensions. For all of them the scaling of the near-extremal Bekenstein-Hawking entropy with the Hawking temperature is in agreement with a statistical description in terms of free massless fields on the world volume.
\end{abstract}

April 1996

* e-mail address: klebanov@puhep1.princeton.edu

* e-mail address: tseytlin@ic.ac.uk. On leave from Lebedev Physics Institute, Moscow. 


\section{Introduction}

During the past few months remarkable progress towards a microscopic understanding of the black hole entropy has taken place. Strominger and Vafa [1] considered a class of 5-dimensional black holes with non-vanishing Bekenstein-Hawking entropy,

$$
S_{B H}=\frac{A}{4 G_{N}},
$$

where $A$ is the volume of the horizon. The entropy of Dirichlet brane states [2, 3] carrying the same set of charges was found to agree with $S_{B H}$ in the limit appropriate for describing macroscopic black holes [1]. This result has been generalized in a number of ways [4, [5].

The direction that is of immediate relevance to our paper is the extension of the D-brane entropy counting to near-extremal black $p$-branes. A system that has been thoroughly investigated in this regard is the dyonic (self-dual) black string in 6 dimensions [4, 60.1 To leading order away from extremality, the Bekenstein-Hawking entropy of the dyonic string agrees with the counting of low-energy D-brane excitations [4, 6, 8]. Another interesting laboratory for near-extremal entropy is the self-dual 3-brane in 10 dimensions [9,10. The feature that the self-dual string in 6 dimensions and the self-dual 3-brane in 10 dimensions share is that these solutions are non-dilatonic: the dilaton field is constant throughout space-time. A divergence of the dilaton field at the horizon, that is common for other $p$-branes, is indeed dangerous because it may lead to a strong coupling problem. For the 3-brane system one finds that the Bekenstein-Hawking and the D-brane definitions of entropy agree on the scaling exponents with respect to mass and charge [9, 10]. The Bekenstein-Hawking entropy of $n$ coincident 3-branes is reproduced by the statistical mechanics of $6 n^{2}$ free massless fermions and bosons in $3+1$ dimensions. On the other hand, at weak coupling the world volume theory appears to have $8 n^{2}$ massless degrees of freedom. While we do not understand the resolution of this puzzle, we will offer some guesses. believe that this 3-brane puzzle will, in the end, find an interesting resolution. In this paper we plan to make similar free field comparisons for other D-brane systems, hoping to get some information about their world volume structure.

1 The extremal limit of the dyonic string solution was found in [7]. Upon compactification on a circle, its 'boosted' equal charge version reduces to the black hole considered in [1].

$2 n$ coincident 3 -branes are described by $\mathcal{N}=4$ supersymmetric $U(n)$ gauge theory at the point where the full non-abelian symmetry is restored. In a sense, at this point monopoles and dyons become massless which may affect the counting of low-energy states. We thank E. Kiritsis for useful discussions on this issue. 
The primary purpose of this paper is to study the near-extremal Bekenstein-Hawking entropy, and its possible statistical interpretation, for a large class of black $p$-branes in various dimensions. One of our results is that a simple interpretation of the entropy in terms of massless fields in $p+1$ dimensions is possible only for the non-dilatonic $p$-branes. In addition to the previously studied systems, these include the 2 - and 5 - branes in $D=11$. We hope, therefore, that our studies will shed some light on the world volume structure of the M-theory branes.

While this paper was in preparation, we received preprint [1] which discuss a general class of black $p$-brane solutions. This class includes the non-dilatonic $p$-branes [12] whose entropy was independently studied by us and which is the primary subject of this paper. For completeness, however, in section 2 we review and interpret the Bekenstein-Hawking entropy of the more general solutions of [1]. We find that there are no dilatonic $p$ branes whose near-extremal entropy may be explained by free massless fields on the world volume. This brings us back to the non-dilatonic $p$-branes which we study in the rest of the paper. The results are especially puzzling for the $D=11 p$-branes. While their Bekenstein-Hawking entropies scale with the Hawking temperature appropriately for the world volume massless field description, the number of such fields is, in general, not an integer. Moreover, for $n$ parallel 5-branes this number grows as $n^{3}$, while for $n$ parallel 2-branes - as $n^{3 / 2}$. We suspect that these exponents may be interpreted in terms of enhanced symmetry of coincident $p$-branes. Coincident 5 -branes are expected to give rise to tensionless strings [13]. Perhaps this is the reason why the number of massless degrees of freedom grows faster than the $n^{2}$ growth associated with restoration of $U(n)$ symmetry found for the D-branes [14].

In section 3 we present our approach to charge quantization which allows us to normalize the Bekenstein-Hawking entropy for systems involving $n$ coincident $p$-branes. In section 4 this is compared with the statistical entropy of $N_{p}$ massless bosons and fermions in $p+1$ dimensions. The numbers $N_{p}$ necessary for complete agreement are deduced and commented on. In section 5 , we conclude the paper with some speculations on 5-branes in $D=12$.

\section{Black p-branes and their entropy}

\subsection{Review of black p-brane solutions}

Black p-brane solutions in various dimensions with one scalar field may be described in a universal way as extrema of the following action (see 115, 16, 12, 17, 18, 11])

$$
S=-\frac{1}{2 \kappa^{2}} \int d^{D} x \sqrt{g}\left[R-\frac{1}{2}(\partial \phi)^{2}-\frac{1}{2(d+1) !} e^{a \phi} F_{d+1}^{2}\right], \quad D=p+d+3 .
$$


A general class of such black p-brane solutions was found recently in [11]. This class generalizes, in particular, the dilatonic solutions of [15,16] to arbitrary $d$ and $p$ not constrained by $N=1$ (see $(2.2)$ below). [11] also presents dilatonic $(a \neq 0)$ generalizations of the non-dilatonic solutions found in [12]. The metrics found in [1] are 'superpositions' of extremal solutions, parametrized by harmonic functions $H$, and the Schwarzschild solutions parametrized by function $f$,

$$
\begin{gathered}
d s^{2}=H^{\alpha}(r)\left(H^{-N}(r)\left[-f(r) d t^{2}+d y_{1}^{2}+\ldots+d y_{p}^{2}\right]+f^{-1}(r) d r^{2}+r^{2} d \Omega_{d+1}^{2}\right), \\
H=1+\frac{r_{-}^{d}}{r^{d}}, \quad f=1-\frac{\mu^{d}}{r^{d}}, \quad r_{-}^{d} \equiv \mu^{d} \sinh ^{2} \gamma \\
\alpha=\frac{N(p+1)}{D-2}, \quad N \equiv 4\left[a^{2}+\frac{2 d(p+1)}{D-2}\right]^{-1} .
\end{gathered}
$$

The extremal limit corresponds to $\mu \rightarrow 0(f \rightarrow 1)$ and $\gamma \rightarrow \infty$ with $\mu^{d} \sinh 2 \gamma$ kept fixed. We are primarily interested in solutions which are supersymmetric in the extremal limit. For such solutions, $N$ is an integer [17, 18]. $N$ may be interpreted as a measure of 'compositeness' of a configuration, i.e. the number of different field strengths or charges that were set equal to each other in reducing the action to the form (2.1) [18,11]. For $N=1,2$ or 3 , the fraction of residual supersymmetry is $1 / 2^{N}$; for $N=4$ it is $1 / 8$ for solutions with $d=1$ (i.e., the configurations which look like black holes upon reduction to $D=4$ ) or $1 / 16$ for solutions with $d=0$.

In addition to the metric, (2.2), the solutions of [11] involve the field strength and the dilaton. Here it is important whether the solution is purely 'magnetic' or dyonic. For purely 'magnetic' solutions,

$$
F_{d+1}=\frac{1}{2} \sqrt{N} d \mu^{d} \sinh 2 \gamma \epsilon_{d+1}, \quad e^{2 \phi}=H^{-a N}
$$

Related dyonic solutions are obtained from the 'magnetic' one by performing a duality transformation on the $d$-form field which leaves the metric invariant but affects the dilaton. We may have dyons if $D=2 p+4$ (i.e. $d=p+1$ ). A well-known example of this is the

3 Our notation differs somewhat from that in [11]. The relation to the radial coordinate used in 15, 16, 12] is the following: $\hat{r}^{d}=r^{d}+r_{-}^{d}=r^{d} H(r), \Delta_{-}(\hat{r})=H^{-1}(r), \Delta_{+}(\hat{r})=H^{-1}(r) f(r)$, $\Delta_{ \pm}(\hat{r})=1-\frac{r_{ \pm}^{d}}{\hat{r}^{d}}, \quad r_{+}^{d}=\mu^{d} \cosh ^{2} \gamma, r_{-}^{d}=\mu^{d} \sinh ^{2} \gamma$ (with $r_{ \pm} \rightarrow r_{0}$ in the extremal limit). When departing from extremality, fixing the charge translates into holding $r_{+} r_{-}$fixed. 
Reissner-Nordstrom black hole in $D=4(d=p+1=1, N=4)$. . For dyons with equal electric and magnetic charge, the dilaton is constant ( $a$ may be set to zero), and 0

$$
F_{p+2}=F_{d+1}=\frac{1}{2 \sqrt{2}} \sqrt{N}(p+1) \mu^{d} \sinh 2 \gamma\left(\epsilon_{p+2}+\epsilon_{p+2}^{*}\right) .
$$

In the special case of $D=2 p+4$ where $p$ is odd, the equal charge dyonic solutions are real self-dual: these are the self-dual string [16,12] in $D=6(d=p+1=2, N=2)$ and the self-dual 3-brane in $D=10$ [15, 19] $(d=p+1=4, N=1)$. We will also be concerned with the 11-dimensional solutions: the fundamental 2-brane [20 $(d=6, p+1=3, N=1)$ and the solitonic 5-brane [21] $(d=3, p+1=6, N=1)$. These solutions are also non-dilatonic simply because there is no dilaton in $D=11$. As shown in [12], for all these non-dilatonic solutions there exist Bogomolnyi bounds relating the mass and charge at extremality. Well-known examples of dilatonic solutions, which will be used in our discussion of the entropy, are the solitonic and the $\mathrm{R}-\mathrm{R}$ charged 5-branes [22,23, 15 in $D=10(d=2, p+1=6, N=1)$.

We shall assume that the internal dimensions $y_{i}$ of a $p$-brane are compactified on a torus with equal periods $L$. Let us first consider the purely 'magnetic' solutions (the discussion of the purely 'electric' case is identical). The charge per unit volume and the ADM mass are given by [24, 16, 11]

$$
\begin{gathered}
q_{p}=\frac{\omega_{d+1}}{2 \sqrt{2} \kappa} \sqrt{N} d \mu^{d} \sinh 2 \gamma \equiv \frac{\omega_{d+1}}{\sqrt{2} \kappa} \sqrt{N} d r_{0}^{d}, \\
M_{p}=\frac{\omega_{d+1}}{2 \kappa^{2}} L^{p} \mu^{d}\left(d+1+N d \sinh ^{2} \gamma\right)=\frac{\sqrt{2} q_{p}}{\kappa \sqrt{N} d} \frac{d+1+N d \sinh ^{2} \gamma}{\sinh 2 \gamma},
\end{gathered}
$$

where $\omega_{d+1}=2 \pi^{\frac{d}{2}+1} / \Gamma\left(\frac{d}{2}+1\right)$ is the volume of a unit $(d+1)$-sphere. For self-dual solutions $(d=p+1)$, e.g. the string in $D=6$ and the 3-brane in $D=10$, the mass is the same while the electric and magnetic charges are each equal to $\frac{1}{\sqrt{2}}$ of $q_{p}$ in (2.7). The $(D-2)$-volume of the horizon, located at $r=\mu$, is found to be

$$
A_{p}=\omega_{d+1} \mu^{d+1} L^{p} H^{\frac{N}{2}}(\mu)=\omega_{d+1}^{-\frac{1}{d}} L^{p}\left(\frac{2 \sqrt{2} \kappa q_{p}}{\sqrt{N} d}\right)^{\frac{d+1}{d}}(\sinh 2 \gamma)^{-\frac{d+1}{d}}(\cosh \gamma)^{N}
$$

4 When embedded into string theory, this is the dyonic black hole with 4 equal (two electric and two magnetic) charges. Though the charges are equal, one needs at least two different (electric and magnetic) vector fields since the $p=0, D=4$ self-duality condition does not have real solutions. This is different from the $p=1, D=6$ and $p=3, D=10$ cases: though formally they may be obtained as self-dual limits of dyonic p-brane solutions, they may be interpreted as intrinsic solutions of theories with self-dual $(p+2)$-field strengths, the self-dual gravity in $D=6$ and type IIB supergravity in $D=10$.

5 The rescaling of the magnetic charge by $1 / \sqrt{2}$ is necessary in order to get the same expression for the metric in the presence of extra electric charge, equal to the magnetic one. 
This volume, and the corresponding Bekenstein-Hawking entropy, $S_{p}=2 \pi A_{p} / \kappa^{2}$, have non-vanishing values in the extremal limit iff

$$
\lambda \equiv \frac{d+1}{d}-\frac{N}{2}=0 .
$$

For supersymmetric $p$-branes (those with integer $N$ ) there are only two solutions of this condition: $d=1, N=4$ (i.e. $p=D-4$ ) and $d=2, N=3$ (i.e. $p=D-5$ ). These respectively correspond to the equal charge dyonic black holes in $D=4$ [25,26, 27] and $D=5$ [1, 28, 29, 30], or to composite higher-dimensional p-branes which reduce to such black holes upon wrapping over compact dimensions. This analysis implies, in particular, that there are no finite entropy extremal black holes in $D=6$ and higher. One may justify this conclusion also by a different argument. Let us try to construct an extremal supersymmetric $D>5$ black holes using the 'boosted' fundamental string solution,

$$
d s^{2}=H^{-1}\left(-d t^{2}+d y^{2}\right)+(d t-d y)^{2}+d x_{i}^{2}, \quad e^{2 \phi}=H^{-1}
$$

and the R-R $p$-brane solution $(p \leq 4)$,

$$
d s^{2}=H^{-1 / 2}\left(-d t^{2}+d y_{n}^{2}\right)+H^{1 / 2} d x_{i}^{2}, \quad e^{2 \phi}=H^{(3-p) / 2},
$$

as the building blocks (solitonic 5-brane has only $S O(4)$ isometry). To get finite entropy, one needs a BPS 'mixture' with the dilaton and all moduli being finite at the horizon. The metric of a black hole in $D$ dimensions should take the form (for all charges, i.e. harmonic functions, set equal)

$$
d s_{D}^{2}=-f(x) d t^{2}+H^{n} r^{2} d \Omega_{D-2} .
$$

Since $H=1+m / r^{D-3}$, to get a finite area of the $r=0$ horizon it is necessary that $n=2 /(D-3)$. This gives $n=2$ in $D=4, n=1$ in $D=5$, but $n=2 / 3$ in $D=6$. While for $n=1,2$ one can build up $H^{n}$ from the $H^{1 / 2}$ factors in the transverse metric of the R-R $p$-branes, this is impossible for $n=2 / 3$. For $D=7$ we need $n=1 / 2$ but the moduli blow up at $r=0$. Thus, it appears that the extreme dyonic black holes in $D=4$ (with 4 charges) and in $D=5$ (with 3 charges) are the only ones which have finite Bekenstein-Hawking entropy.

\footnotetext{
6 Similar conclusion about the non-existence of supersymmetric extremal $D>5$ black holes with finite area of the horizon was reached by M. Cvetič and D. Youm (private communication) who constructed general non-extreme rotating $\mathrm{BH}$ solutions in $D \geq 6$.
} 


\subsection{The Bekenstein-Hawking entropy of near-extremal black p-branes}

Given that all the $1 / 2$ and $1 / 4$ supersymmetric extremal p-branes have zero entropy, it is of interest to study their near-extremal expressions for the entropy, generalizing previous discussions of the $D=6$ dyonic string [6] and the $D=103$-brane [9]. Our eventual goal is to interpret the leading near-extremal corrections to the black $p$-brane mass and entropy as the energy and entropy of some weakly interacting effective massless degrees of freedom on the $p$-brane world volume. In $D \leq 10$ the degrees of freedom are undoubtedly those of the open strings in the D-brane description of the R-R charged $p$-branes. In the M-theory they are presumably related to closed strings inside a 2-brane [31] or a 5-brane [32]. Thus, we expect our comparison of entropy to be a new source of information about the M-theory.

Let us expand the $p$-brane mass, (2.8), for fixed $q_{p}$ near extremality (for large $\gamma$ )

$$
\begin{aligned}
M_{p}=M_{p 0}\left(1+\frac{\delta M_{p}}{M_{p 0}}\right), \quad M_{p 0} & =\sqrt{N} \frac{q_{p}}{\sqrt{2} \kappa} L^{p}=\frac{\omega_{d+1}}{2 \kappa^{2}} d N r_{0}^{d} L^{p}, \\
\frac{\delta M_{p}}{M_{p 0}} & =\frac{4 \lambda}{N} e^{-2 \gamma},
\end{aligned}
$$

where $\lambda$ is defined in (2.10). Assuming $\lambda \geq 0$, the area of the horizon, (2.9), becomest

$$
\begin{aligned}
& A_{p}=4^{\lambda} \omega_{d+1}^{-\frac{1}{d}} L^{p}\left(\frac{\sqrt{2} \kappa q_{p}}{\sqrt{N} d}\right)^{\frac{d+1}{d}} e^{-2 \lambda \gamma} \\
& =\omega_{d+1}^{-\frac{1}{d}} L^{p}\left(\frac{\sqrt{2} \kappa q_{p}}{\sqrt{N} d}\right)^{\frac{d+1}{d}}\left(\frac{N \delta M_{p}}{\lambda M_{p 0}}\right)^{\lambda} .
\end{aligned}
$$

Interpreting $\delta M_{p}$ as the energy $E$, and using (2.11), we obtain the following expression for the entropy,

$$
S_{p}=\frac{2 \pi A_{p}}{\kappa^{2}}=4 \pi \omega_{d+1}^{-\frac{1}{d}} L^{p(1-\lambda)} d^{-\frac{d+1}{d}} \lambda^{-\lambda}(\sqrt{2} \kappa)^{\frac{2}{d}-\frac{N}{2}}\left(\frac{q_{p}}{\sqrt{N}}\right)^{\frac{N}{2}} E^{\lambda}
$$

Remarkably, the dependence of the entropy on the charge looks universal - each of the $N$ 'constituent' $1 / 2$ supersymmetric objects making up the $p$-brane contributes a factor of $\sqrt{q_{p}}$.

Employing the thermodynamical relation $d E=T d S$ we find the corresponding Hawking temperature,

$$
T^{-1}=4 \pi \omega_{d+1}^{-\frac{1}{d}}(\sqrt{2} \kappa)^{\frac{2}{d}-\frac{N}{2}} d^{-\frac{d+1}{d}} \lambda^{1-\lambda} L^{p}\left(\frac{q_{p}}{\sqrt{N}}\right)^{\frac{N}{2}} E^{\lambda-1}
$$

7 For $\lambda=0$ the expression for the entropy given below applies to the extremal case. In this special sitation we ignore the subleading corrections to the entropy. 
Notice that the temperature does not depend on the energy if $\lambda=1$. This happens if $N=2 / d$, i.e. if $d=1, N=2$ and also if $d=2, N=1$. The latter case $(p=D-5)$ corresponds to the 5 -brane in $D=10$ or the fundamental string in $D=6$. Thus, for the $D=105$-brane we get a surprisingly simple expression for the entropy

$$
S_{5}^{(10)}=\left(\sqrt{2} \kappa q_{5}\right)^{1 / 2} E
$$

Note that the explicit dependence on the volume, and all the factors of $\pi$, have canceled out. For the R-R charged 5-brane the string coupling goes to zero at the horizon. Perhaps the fact that the entropy is linear in the energy may be interpreted as the absence of thermodynamical equilibrium in this system due to the vanishing coupling on the world volume.

For $\lambda \neq 1$ we may express the entropy in terms of the charge and the temperature,

$$
S_{p} \propto L^{p} q_{p}^{\frac{N}{2(1-\lambda)}} T^{\frac{\lambda}{1-\lambda}}
$$

It is interesting to identify the cases where the Bekenstein-Hawking entropy scales in the same way as the entropy of an ideal gas of massless particles in $p$ dimensions (the gas of massless modes on the world volume), i.e. when $S_{p} \sim T^{p}$. The condition $\frac{\lambda}{1-\lambda}=p$ is equivalent to $p+1=2 d /(N d-2)$. It follows that $a=0$, i.e. that the dilaton is constant. Then

$$
N=\frac{2}{p+1}+\frac{2}{d}=\frac{2(D-2)}{(p+1)(D-3-p)}, \quad \lambda=\frac{p}{p+1} .
$$

We conclude that for all the non-dilatonic p-branes the entropy has the natural massless ideal gas scaling, $S_{p} \sim L^{p} T^{p}$. This is a reasonable conclusion: only if the coupling is wellbehaved may we hope to reproduce black $p$-brane thermodynamics by a simple weakly interacting ensemble. The condition (2.18) is very restrictive in the supersymmetric cases: if $N=12$ (2.18) is satisfied only for $p=2$ and $p=5$ in $D=11$ and for $p=3$ in $D=10$; if $N=2$ the only solution is $p=1$ in $D=6$.

In what follows we shall specialize to non-dilatonic p-branes [12 (2.18), but keep $N$ arbitrary. Then

$$
S_{p}=\frac{4 \pi \omega_{d+1}}{2 \kappa^{2}}\left(\frac{4 \pi}{d}\right)^{p}\left(\frac{\sqrt{2} \kappa}{\omega_{d+1} \sqrt{N} d} q_{p}\right)^{\frac{D-2}{D-3-p}} L^{p} T^{p}
$$

or, equivalently,

$$
S_{p}=\frac{4 \pi \omega_{d+1} r_{0}^{d+1+p}}{2 \kappa^{2}}\left(\frac{4 \pi}{d}\right)^{p} L^{p} T^{p} .
$$

The charge thus enters in the power 2 for the self-dual string in $D=6$ and 3 -brane in $D=10$, in the power 3 for the 5 -brane in $D=11$, and the power $3 / 2$ for the 2 -brane in 
$D=11$. For the first two of these, the self-dual p-branes $(d=p+1, N=4 /(p+1))$, the expression for the entropy reduces to

$$
S_{p}=\frac{1}{2 \omega_{p+2}}\left(\frac{4 \pi}{p+1}\right)^{p+1} q_{p}^{2} L^{p} T^{p} .
$$

\section{Charge quantization conditions}

To carry out a detailed comparison of the above expressions for the BekensteinHawking entropy with statistical mechanics, we need to decide how the $p$-brane charge is quantized. In the case when both elementary and solitonic p-branes exist in the same theory the corresponding 'electric' and 'magnetic' charges satisfy the quantization condition $(\tilde{d}=p+1)[33$

$$
e_{\tilde{d}} g_{d}=2 \pi m
$$

where $m$ is an integer. For the elementary charges this condition is typically satisfied with $m=1$.

In the dyonic case, the quantization condition becomes $e_{d^{\prime}} g_{d}^{\prime}-e_{d^{\prime}}^{\prime} g_{d}=2 \pi m$. This does not a priori fix the value of $e_{p+1}=g_{p+1}=q_{p}$ in self-dual case. Here we need to envoke some extra information about how the quantum theory is actually defined. For example, in the case of the dyonic string in $D=6$ one may consider it being a special NS-NS solitonic string solution with quantization of charges being fixed by fundamental string world-sheet considerations (e.g. quantization of magnetic charge is fixed by its relation to the WZW term in the string action [34] or the level of the $S U(2)$ WZW model). The resulting charge quantization (which agrees with the one used in [6]) might differ from the one that applies to the genuine interacting $D=6$ self-dual string. In the 3 -brane case the unit of charge is fixed by interpreting it as a $D$-brane configuration in type IIB superstring [9].

Since for $D=2 p+4$ the electric and the magnetic $p$-branes are interchanged by the weak-strong coupling duality, we assume that the elementary electric and magnetic charges are equal. Using the quantization condition (3.1) with $m=1$, we find that the elementary unit of charge is $\sqrt{2 \pi}$. Thus, the possible values of charge are $q_{p}=\sqrt{2 \pi} n$, where $n$ is the integer equal to the number of coincident $p$-branes. We believe that this quantization rule is correct for dyonic strings in $D=6$, and, in particular, for the special case of equal charges (as mentioned above, this is justified by reference to fundamental string theory). For dyonic systems with equal charge, (2.21) becomes

$$
S_{p}=\frac{\pi}{\omega_{p+2}}\left(\frac{4 \pi}{p+1}\right)^{p+1} n^{2} L^{p} T^{p} .
$$

For example, for the $D=40$-brane and the $D=6$ 1-brane we find, respectively,

$$
S_{0}=\pi n^{2}, \quad S_{1}^{\text {dyonic }}=2 \pi n^{2} L T .
$$


This result for the string agrees with the statistical count of entropy based on D-branes [6].

At the same time, the $D$-brane interpretation of 3 -branes implies [9] a quantization condition which is different by a factor of 2 (this 'compensates' for $1 / \sqrt{2}$ factor in the expression for the charge (2.7) in the dyonic case)

$$
q_{p}^{2}=\pi n
$$

Then

$$
S_{p}=\frac{\pi}{2 \omega_{p+2}}\left(\frac{4 \pi}{p+1}\right)^{p+1} n^{2} L^{p} T^{p}
$$

We believe that this result is correct both for the self-dual 3-brane and for the 'truly self-dual' string,

$$
S_{1}^{\text {self-dual }}=\pi n^{2} L T, \quad S_{3}=\frac{\pi^{2}}{2} n^{2} L^{3} T^{3} .
$$

Thus, there may be a subtle difference between the equal charge case of the dyonic string, considered in [6], and the 'truly self-dual' string. Clearly, this deserves further investigation.

Let us also note that, in addition to the obvious similarities, there are also obvious differences between dyonic strings in $D=6$ and 3-branes in $D=10$. For the former, the tension is given in terms of the integral electric and magnetic charges, $P$ and $Q$, by $\left(e^{-\phi_{0}} P+e^{\phi_{0}} Q\right) / 2 \pi \alpha^{2}$. For the equal-charge dyonic string, $P=Q$ and $e^{\phi_{0}}=1$, which implies that there is no freedom to adjust the string coupling. This is also consistent with the absence of the dilaton field in the six-dimensional self-dual gravity.

For the self-dual 3-brane there is no 'dyonic' generalization with $P \neq Q$ because it is stabilized by the self-dual 5-form. Another apparent difference is that the dilaton may assume any constant value. As we remarked earlier, it is not obvious, however, that there is a limit where the world volume theory of multiple 3-branes is truly weakly coupled. The charge quantization subtleties that we have discussed suggest that, even when classical solutions are identical, there may be a difference between 'truly self-dual' objects and equal-charge dyonic objects.

Let us now turn to the case of our main interest - $p$-branes in $D=11$ supergravity or 'M-theory'. We shall assume that there exist both the fundamental 2-branes and the solitonic 5-branes with their unit charges related by

$$
q_{2} q_{5}=2 \pi
$$

The unit 2-brane charge is fixed in terms of the tension $T_{2}$ in the elementary 2-brane action, which plays the role of a source in supergravity equations of motion,

$$
q_{2}=\sqrt{2} \kappa T_{2}
$$


This normalization is consistent with the fundamental string one. Assuming that 2-brane is wrapped around 2-torus with periods $L$ we may use double dimensional reduction [35] to relate the membrane solution to the fundamental string solution [36]. It is easy to see that the string and membrane tensions, $T_{1}=1 / 2 \pi \alpha^{\prime}$ and $T_{2}$, are related by

$$
T_{2}=T_{1} / L, \quad T_{2} \kappa^{2}=T_{1} \kappa_{10}^{2}
$$

where the 10-dimensional gravitational constant is expressed in terms of the 11-dimensional one by $\kappa_{10}^{2}=\kappa^{2} / L$. If the string is wrapped $n$ times around the 9 -th dimension the coefficient in the corresponding harmonic function is $r_{-}^{6}=\frac{2 \kappa_{10}^{2}}{6 \omega_{7}} n T_{1}=\frac{2 \kappa^{2}}{6 \omega_{7}} n T_{2}$.

In order to determine $T_{2}$ in terms of $\kappa$ we need one more input. We will use the fact that the double dimensional reduction of the M-theory 5-brane gives the Dirichlet 4-brane of the type IIA theory,

$$
T_{5} \kappa^{2}=T_{4} \kappa_{10}^{2}
$$

The unit 5-brane charge is related to $T_{5}$ by $q_{5}=\sqrt{2} \kappa T_{5}$. Using $\kappa_{10}=g \alpha^{\prime 2}$, eqs. (3.7)(3.10), and the known D-brane tension [3], $\kappa_{10} T_{4}=\left(2 \sqrt{\pi \alpha^{\prime}}\right)^{-1}$, we solve for $\kappa$ in terms of the ten-dimensional quantities,

$$
\kappa^{2}=\frac{g^{3} \alpha^{\prime 9 / 2}}{4 \pi^{5 / 2}}
$$

Our argument further determines the charges and tensions of the M-branes. In particular, 8

$$
T_{2}^{3}=\frac{2 \pi^{2}}{\kappa^{2}}
$$

Thus, the 2-brane charge takes on the following values,

$$
q_{2}=n \sqrt{2}\left(2 \kappa \pi^{2}\right)^{1 / 3}
$$

The quantization condition (3.1) fixes the possible values of the 5-brane charge to be

$$
q_{5}=\frac{2 \pi n}{\sqrt{2} \kappa T_{2}}=n \sqrt{2}\left(\frac{\pi}{2 \kappa}\right)^{1 / 3} .
$$

Substituting these expressions into the entropy (2.19), we find for $n$ coincident 2-branes in $D=11\left(p=2, d=6, \omega_{7}=\pi^{4} / 3\right)$,

$$
S_{2}^{(11)}=2^{7 / 2} 3^{-3} \pi^{2} n^{3 / 2} L^{2} T^{2} .
$$

8 In ref. [37] it was argued on the basis of membrane path integrals that $\kappa^{2} T_{2}^{3}=\pi^{2} / m_{0}$. The quantity $m_{0}$ was left ambiguous, however. Our line of reasoning based on relation to $D=10$ string theory fixes the value of $m_{0}$ to be $1 / 2$. It would be interesting to find an intrinsic M-theory argument which explains why $\kappa^{2} T_{2}^{3}=2 \pi^{2}$ is the correct quatization condition. 
For $n$ coincident 5 -branes in $D=11\left(p=5, d=3, \omega_{4}=8 \pi^{2} / 3\right)$,

$$
S_{5}^{(11)}=2^{7} 3^{-6} \pi^{3} n^{3} L^{5} T^{5}
$$

\section{Statistical entropy of massless modes on $p$-branes and comparison to the Bekenstein-Hawking entropy}

Let us take a broader point of view than the one suggested by the D-brane picture (cf. [38, [4,6,9] ). We simply ask whether it is possible to understand the entropy of near-extremal non-dilatonic black p-branes in terms of the degeneracy of some weakly interacting massless $p$-brane excitation modes. The corresponding free energy and entropy will be that of an ideal gas of massless particles in $p$ spatial dimensions. The number of boson and fermion fields is, of course, determined by the detailed properties of a particular $p$-brane.

\subsection{Massless ideal gas}

The free energy of massless Bose gas in $p>0$ spatial dimensions identified with periods $L$ is, in the thermodynamic limit of large $L$,

$$
\begin{gathered}
-F_{p} / T=\ln Z_{p}=L^{p} \int \frac{d^{p} k}{(2 \pi)^{p}} \ln \left(1-e^{-|k| / T}\right)=c_{p} L^{p} T^{p}, \\
c_{p}=(2 \pi)^{-p} \omega_{p-1}(p-1) ! \zeta(p+1) .
\end{gathered}
$$

The corresponding entropy and energy are given by

$$
S_{p}=-\frac{\partial F_{p}}{\partial T}=(p+1) c_{p} L^{p} T^{p}, \quad E=T^{2} \frac{\partial \ln Z_{p}}{\partial T}=p c_{p} L^{p} T^{p+1} .
$$

The statistical treatment of a massless fermion field is analogous, with the conclusion that one real fermion contributes the entropy equal to $\left(1-2^{-p}\right)$ times that of a massless boson. If the extremal $p$-brane preserves some supersymmetry, then the number of massless bosons should equal the number of massless fermions. Denoting this number by $N_{p}$, we find that the total statistical entropy is

$$
S_{p}=b_{p} L^{p} T^{p}, \quad b_{p}=N_{p}\left[1+\left(1-2^{-p}\right)\right](p+1) c_{p}
$$

For the particular cases of a string, a 3-brane, and a 5-brane we find

$$
b_{1}=\frac{\pi}{2} N_{1}, \quad b_{3}=\frac{\pi^{2}}{12} N_{3}, \quad b_{5}=\frac{\pi^{3}}{40} N_{5} .
$$

For the 2-brane, on the other hand, we find $b_{2}=\frac{7}{8 \pi} \zeta(3) N_{2}$. In general, the coefficients $b_{p}$ contain $\zeta(p+1)$, which for even $p$ are not expressible in terms of $\pi$. Thus, for even $p$ 
there cannot be a detailed agreement between the Bekenstein-Hawking and the statistical entropy. This may be related to the fact that the even $p$ solutions are singular, while the non-dilatonic odd $p$ solutions are not [12]. It is nevertheless intriguing that for the 2-brane in 11 dimensions the scaling of the entropy with respect to the temperature does agree with the massless statistical mechanics.

For the non-dilatonic $p$-branes with $p$ odd, we hope to find a more detailed agreement. Indeed, comparing (4.4) with the Bekenstein-Hawking entropies calculated in section 3, we find that the powers of $\pi$ match perfectly. For the equal-charge (self-dual) case of the dyonic string in $D=6$, we need $N_{1}=4 n^{2}$ to achieve perfect agreement. This is indeed the number predicted by the D-brane counting of [6]. The factor of 4 may be thought of as the number of transverse modes of a string in 6 dimensions. The advantage of our approach to charge quantization and the normalization of the entropy is that, although somewhat heuristic, it could be carried out without any knowledge of the D-branes.

To achieve perfect agreement for the self-dual 3-brane, we need $N_{3}=6 n^{2}$. As suggested in [9], it is tempting to think of the factor 6 as the number of transverse modes of a 3-brane in 10 dimensions. It is remarkable that the $n^{2}$ growth is in agreement with the enhanced $U(n)$ symmetry of $n$ coincident 3-branes [9]. Naively, the number of massless field modes is $8 n^{2}$, but massless monopoles and dyons are, in a sense, also present. Perhaps $8 n^{2}$ is reduced to $6 n^{2}$ by a confinement mechanism.

\subsection{The 5-brane in $D=11$}

For $n$ coincident $D=11$ 5-branes we need

$$
N_{5}=2^{10} 3^{-6} 5 n^{3}
$$

to achieve perfect agreement between the statistical and the Bekenstein-Hawking entropies. While we certainly do not have enough knowledge about the world volume theory to derive this number of massless degrees of freedom, we have several comments.

The most notable feature, which does not depend on the details of how the charge is quantized, is the $n^{3}$ growth of $N_{5}$. This is consistent with the idea of enhanced symmetry for coincident 5-branes, but grows faster than the dimension of any Lie group. Perhaps this faster growth than the one found for D-brane systems is related to the appearance of tensionless strings [13,39]. Based on our entropy considerations, we speculate that the enhanced symmetry in M-theory is a new kind of phenomenon that needs to be understood better.

Unlike in the 1-brane and 3-brane cases, the number of massless degrees of freedom is in general fractional, but becomes integer when $n$ is a multiple of 9 . It is conceivable that the fractional effective number of massless fields is a regularized version of the infinite number of massless fields which appear when the closed string in $5+1$ dimensions becomes 
tensionless. Furthermore, the world volume theory is not expected to be weakly coupled, which further complicates matters.

If we look for a pattern in the entropy results, it seems that the correct factor might simply be the number of transverse modes. What could be the possible justification for ignoring the longitudinal modes, which in the D-brane description are the world volume gauge fields? While we do not know the answer, we note that the gauge modes are connected with the antisymmetric tensor fields in the bulk. Indeed, the world volume gauge fields may be thought of as gauge transformation parameters for $B_{\mu \nu}$. For this reason it may be redundant to count these modes. Of course, we hope that there is a good dynamical justification for excluding them. For the 5 -brane in $D=11$ there are 5 transverse modes, and by analogy we might have expected that $N_{5}=5 n^{3}$. The extra factor we are finding, $2^{10} 3^{-6}$, is numerically close to 1 and may one day find an explanation.

While there are many puzzles remaining, we feel that the close correspondence between the Bekenstein-Hawking and the statistical entropies for a 5-brane in 11 dimensions are unlikely to be a mere coincidence. Both the scaling with respect to $T$ and the power of $\pi$ match, suggesting the presence of massless fields on the world volume. We hope that the detailed counting teaches us something about the effective action for the world volume theory.

\section{Concluding remarks}

In this paper we discussed the entropies of several non-dilatonic $p$-brane systems: the dyonic string in $D=6$, the self-dual 3 -brane in $D=10$, and the 2 - and 5 -branes in $D=11$. For all these cases, and especially for the odd-dimensional branes, there is a close correspondence between the Bekenstein-Hawking entropy and the statistical entropy of massless fields on the world volume.

We cannot resist taking this one step further and offering some vague speculations about the 5 -brane in $D=12$. Although there is no known supergravitySubst for $D>11$, there has been some recent evidence in favor of the existence of a 12-dimensional theory 40,41,31. In particular, the important role played by the 3-brane of type IIB theory suggests a possibility of its 12-dimensional reformulation 442. Since the elementary string in $D=10$, the elementary 2-brane in $D=11$, and the elementary 3-brane in $D=12$ are all dual to the 5-brane, this object seems to have a special role. If we assume that there is a non-dilatonic 5-brane solution in $D=12$, and apply a very heuristic charge quantization procedure based on comparing it with the 3-brane, we find that the Bekenstein-Hawking entropy is quantized in units of

$$
S_{5}^{(12)}=\frac{6}{5} \pi^{3} L^{p} T^{p}
$$

Amusingly, this corresponds to $N_{5}=6 \times 8$ degrees of freedom on the world volume. One may speculate that 6 is due to the number of transverse modes of the 5-brane in 12 dimensions, while 8 is related to the number of world sheet degrees of freedom of the self-dual string. In any case, unlike for $D=11$, we seem to find an integer number of massless fields on the world volume. While at the moment we can offer little more than

this kind of numerology, we believe that further work will shed more light on the dynamics of $p$-branes in the hypothetical 12-dimensional theory. 


\section{Acknowledgements}

We thank C. Callan, R. Dijkgraaf, E. Kiritsis and E. Verlinde for interesting discussions. I.R.K. was supported in part by DOE grant DE-FG02-91ER40671, the NSF Presidential Young Investigator Award PHY-9157482, and the James S. McDonnell Foundation grant No. 91-48. A.A.T. would like to acknowledge the support of PPARC, ECC grant SC1*-CT92-0789 and NATO grant CRG 940870. 


\section{References}

[1] A. Strominger and C. Vafa, HUTP-96-A002, hep-th/9601029.

[2] J. Dai, R.G. Leigh, and J. Polchinski, Mod. Phys. Lett. A4 (1989) 2073; R.G. Leigh, Mod. Phys. Lett. A4 (1989) 2767.

[3] J. Polchinski, Phys. Rev. Lett. 75 (1995) 4724, hepth/9510017.

[4] C.G. Callan and J.M. Maldacena, PUPT-1591, hep-th/9602043.

[5] J.C. Breckenridge, R.C. Myers, A.W. Peet and C. Vafa, HUTP-96-A005, hepth/9602065.

[6] G.T. Horowitz and A. Strominger, hep-th/9602051.

[7] M.J. Duff, S. Ferrara, R.R. Khuri and J. Rahmfeld, Phys. Lett. B356 (1995) 479, hep-th/9506057.

[8] J.C. Breckenridge, D.A. Lowe, R.C. Myers, A.W. Peet, A. Strominger and C. Vafa, hep-th/9603078.

[9] S.S. Gubser, I.R. Klebanov and A.W. Peet, hep-th/9602135.

[10] A. Strominger, unpublished notes.

[11] M.J. Duff, H. Lü and C.N. Pope, hep-th/9604052.

[12] G.W. Gibbons, G.T. Horowitz and P.K. Townsend, Class. Quant. Grav. 12 (1995) 297, hep-th/9410073.

[13] A. Strominger, hep-th/9512095.

[14] E. Witten, hep-th/9510135.

[15] G.T. Horowitz and A. Strominger, Nucl. Phys. B360 (1991) 197.

[16] M.J. Duff and J.X. Lu, Nucl. Phys. B416 (1994) 301, hep-th/9306052.

[17] H. Lü, C.N. Pope, E. Sezgin and K.S. Stelle, Nucl. Phys. B276 (1995) 669, hepth/9508042.

[18] H. Lü and C.N. Pope, hep-th/9512012.

[19] M.J. Duff and J.X. Lu, Phys. Lett. B273 (1991) 409.

[20] M.J. Duff and K.S. Stelle, Phys. Lett. B253 (1991) 113.

[21] R. Güven, Phys. Lett. B276 (1992) 49.

[22] M.J. Duff and J.X. Lu, Nucl. Phys. B354 (1991) 141.

[23] C.G. Callan, J.A. Harvey and A. Strominger, Nucl. Phys. B359 (1991) 611.

[24] J.X. Lu, Phys. Lett. B313 (1993) 29.

[25] R. Kallosh, A. Linde, T. Ortín, A. Peet and A. van Proeyen, Phys. Rev. D46 (1992) 5278.

[26] M. Cvetič and D. Youm, Phys. Rev. D53 (1996) 584, hep-th/9507090.

[27] M. Cvetič and A.A. Tseytlin, Phys. Lett. B366 (1996) 95, hep-th/9510097.

[28] A.A. Tseytlin, Mod. Phys. Lett. A11 (1996) 689, hep-th/9601177.

[29] M. Cvetič and D. Youm, hep-th/9603100.

[30] G.T. Horowitz, J.M. Maldacena and A. Strominger, hep-th/9603109. 
[31] D. Kutasov and E. Martinec, hep-th/9602049.

[32] R. Dijkgraaf, E. Verlinde and H. Verlinde, hep-th/9603126; hep-th/9604055.

[33] R. Nepomechie, Phys. Rev. D31 (1985) 1921; C. Teitelboim, Phys. Lett. B167 (1986) 69.

[34] R. Rohm and E. Witten, Ann. Phys. 170 (1986) 454.

[35] M.J. Duff, P.S. Howe, T. Inami and K.S. Stelle, Phys. Lett. B191 (1987) 70.

[36] A. Dabholkar and J.A. Harvey, Phys. Rev. Lett. 63 (1989) 478; A. Dabholkar, G.W. Gibbons, J.A. Harvey and F. Ruiz-Ruiz, Nucl. Phys. B340 (1990) 33.

[37] M.J. Duff, J.T. Liu and R. Minasian, Nucl. Phys. B452 (1995) 261, hep-th/9506126.

[38] S. Das and S. Mathur, hep-th/9601152.

[39] E. Witten, Contribution to Strings '95, hep-th/9507121.

[40] C. Hull, hep-th/9512181.

[41] C. Vafa, hep-th/9602022.

[42] A.A. Tseytlin, Nucl. Phys. B469 (1996) 12, hep-th/9602064. 